\title{
Cetrimonium Bromide Inhibits Cell Migration and Invasion of Human Hepatic SK-HEP-1 Cells Through Modulating the Canonical and Non-canonical TGF- $\beta$ Signaling Pathways
}

\author{
TSAI-KUN WU ${ }^{1,2,3 *}$, CHUNG-HUNG CHEN ${ }^{4 *}$, YING-RU PAN $^{5}$, \\ CHING-WEN HU ${ }^{6}$, FU-MEI HUANG ${ }^{7}$, JER-YUH LIU $^{8,9}$ and CHIA-JEN LEE ${ }^{5,10}$ \\ ${ }^{1}$ Division of Renal Medicine, Tungs' Taichung Metroharbor Hospital, Taichung, Taiwan, R.O.C.; \\ ${ }^{2}$ Department of Nutrition, Master Program of Biomedical Nutrition, Hungkuang University, Taichung, Taiwan, R.O.C.; \\ ${ }^{3}$ Department of Nursing, Jen-Teh Junior College of Medicine, Nursing and Management, Miaoli, Taiwan, R.O.C.; \\ ${ }^{4}$ Department of Gastroenterology, Chang Bing Show Chwan Memorial Hospital, Changhua, Taiwan, R.O.C.; \\ ${ }^{5}$ Department of Medical Research, Tungs' Taichung Metroharbor Hospital, Taichung, Taiwan, R.O.C.; \\ ${ }^{6}$ Department of Nursing, Tungs' Taichung Metroharbor Hospital, Taichung, Taiwan, R.O.C.; \\ ${ }^{7}$ Operating Theatre, Chung Shan Medical University Hospital, Taichung, Taiwan, R.O.C.; \\ ${ }^{8}$ Center for Molecular Medicine, China Medical University Hospital, Taichung, Taiwan, R.O.C.; \\ ${ }^{9}$ Graduate Institute of Biomedical Sciences, China Medical University, Taichung, Taiwan, R.O.C.; \\ ${ }^{10}$ Department of Rehabilitation, Jen-Teh Junior College of Medicine, Nursing and Management, Miaoli, Taiwan R.O.C.
}

\begin{abstract}
Background/Aim: Cetrimonium bromide (CTAB), a quaternary ammonium surfactant, is an antiseptic agent against bacteria and fungi. However, the mechanisms by which its pharmacological actions affect epithelialmesenchymal transition (EMT) in hepatocellular carcinoma (HCC) cells, such as adenocarcinoma in SK-HEP-1 cells, have not been investigated. We, thereby, investigated whether $C T A B$ inhibits cellular mobility and invasiveness of human hepatic adenocarcinoma in SK-HEP-1 cells. Materials and Methods: SK-HEP-1 cells were treated with CTAB, and subsequent migration and invasion were measured by wound healing and transwell assays. Protein expression was detected by immunoblotting analysis. Results: Our data revealed that treatment of SK-HEP-1 cells with CTAB altered their mesenchymal spindle-like morphology. CTAB exerted inhibitory effects on the migration and invasion of SK-HEP-I cells dose-dependently, and reduced protein levels of matrix metalloproteinase-2 (MMP-2), MMP-9, snail, slug, twist,
\end{abstract}

*These Authors contributed equally to this study.

Correspondence to: Chia-Jen Lee, Department of Medical Research, Tungs' Taichung Metroharbor Hospital, No.699, Sec. 8, Taiwan Blvd., Taichung 435, Taiwan, R.O.C. Tel: +886 930059075, e-mail: chiajenlee54@gmail.com

Key Words: Cetrimonium bromide (CTAB), hepatic cancer, SKHEP-1, transforming growth factor- $\beta$ (TGF- $\beta$ ). vimentin, fibronectin, N-cadherin, Smad2, Smad3, Smad4, phosphoinositide-3-kinase (PI3K), p-PI3K, Akt, p-Akt, $\beta$ catenin, mammalian target of rapamycin (mTOR), p-mTOR, p-p70S6K, p-extracellular signal-regulated kinases (ERK)1/2, p-p38 mitogen-activated protein kinase (MAPK) and p-c-Jun $N$-terminal kinase $(J N K)$, but increased protein levels of tissue inhibitor matrix metalloproteinase-1 (TIMP-1), TIMP-2, claudin-1 and p-GSK3 $\beta$. Based on these observations, we suggest that CTAB not only inhibits the canonical transforming growth factor- $\beta$ (TGF- $\beta$ ) signaling pathway though reducing SMADs (an acronym from the fusion of Caenorhabditis elegans Sma genes and the Drosophila Mad, Mothers against decapentaplegic proteins), but also restrains the non-canonical TGF- $\beta$ signaling including MAPK pathways like ERK1/2, p38 MAPK, JNK and PI3K. Conclusion: CTAB is involved in the suppression of TGF- $\beta$ mediated mesenchymal phenotype and could be a potent medical agent for use in controlling the migration and invasion of hepatic adenocarcinoma.

Liver cancer, also known as hepatic cancer, accounts for more than 4.7 percent of all cancer cases and was ranked as the third highest mortality cause among the world's most common cancers in 2018 (8.2\%) (1). Liver contain parenchymal and non-parenchymal cells (NPCs) (2). Hepatocytes are the major parenchymal cells and constitute about $80 \%$ of the liver population. Parenchymal liver cancer can be classified into two major types: i) hepatocellular carcinoma (HCC) and ii) hepatoblastoma. Non-parenchymal 
liver cells can be categorized into numerous types, including biliary epithelial cells, Kupffer cells (KCs), hepatic stellate cells (HSCs), dendritic cells (DCs) and liver sinusoidal endothelial cells (LSECs). Non-parenchymal liver cancer can also come from their respective structures within the liver, such as the bile duct, blood vessels, immune cells and LSECs. Cancers derived from non-parenchymal liver cancer of the bile duct are called cholangiocarcinoma and cholangiocellular cystadenocarcinoma. Tumors originating from blood vessels are called angiosarcoma, hemangioendothelioma, embryonal sarcoma and fibrosarcoma. Adenocarcinoma and lymphomas are classified as rarely malignant tumors arising from nonparenchymal liver cancer of LSECs and immune cells respectively. The prognosis for liver adenocarcinoma is poor due to the greater tendency for vascular invasion, metastasis and recurrence (3). The SK-HEP-1 cell line, used in this study, originating from LSECs, has been derived from the ascitic fluid of a 52-year-old male Caucasian with hepatic adenocarcinoma (4).

Exploring the pathogenesis of liver cancer, studies have shown that metastasis is the major cause of cancer-related mortality $(1,5)$. However, the process of metastasis remains one of the extremely mysterious fields in cancer. In general, the metastatic spread of cancer cells have to be accompanied by resultant changes in the physiological state of the cells, including: i) the increase in cellular motility and invasion, ii) the destruction of cell-cell interactions, iii) the expansion of cell-matrix adhesion, and iv) the degradation of the extracellular matrix (ECM) (6). The first step of the metastatic process is invasion. This includes the step of epithelial to mesenchymal transition (EMT), and subsequently the mesenchymal to epithelial transition (MET). The initiation of EMT exacerbates motility and invasiveness of many cell types and is supposed to be the prerequisite for tumor infiltration and metastasis (7). Therefore, inhibition of EMT of cancer cells is an important mechanism for preventing cancer progression.

In this study, cetrimonium bromide (CTAB, $[(\mathrm{C} 16 \mathrm{H} 33) \mathrm{N}(\mathrm{CH} 3) 3] \mathrm{Br})$ was identified as an effective compound against SK-HEP-1 cell migration by screening the small-molecule database with a high throughput cellular screening system. CTAB is classified as a compound of quaternary ammonium and is widely used as an antiseptic agent against bacteria and fungi (8). Quaternary ammonium derivatives also include the benzethonium chloride and dequalinium chloride. Both of them are resistant to cancer by inhibiting some aspect of mtDNA synthesis (8-10). Other derivatives of quaternary ammonium conjugated with chlorambucil and melphalan have also exhibited significant cytotoxicity against chondrosarcoma (11). In addition, CTAB has been shown to be cytotoxic against human head and neck cancer (HNC) cell lines through a mitochondria-mediated apoptosis pathway (12). However, it is still unknown whether the cytotoxic effects of CTAB, including its anti- viability, anti-proliferative and anti-migration action in liver cancer. In the present study, we determined whether CTAB could inhibit the migration and invasion of the SK-HEP-1 hepatic adenocarcinoma cells. We also investigated the effects of CTAB on molecular changes in SK-HEP-1 cells in terms of cancerous EMT. Finally, we assessed the antimetastatic effects of CTAB on EMT in SK-HEP-1cells and its possible molecular mechanism(s) of action.

\section{Materials and Methods}

Chemicals and antibodies. CTAB was purchased from Sigma (St. Louis, MO, USA). TGF- $\beta 1$ was purchased from PeproTech (Rocky Hill, NJ, USA). Cell proliferation reagent WST-1was purchased from BioVision (Milpitas, CA, USA). Radio immunoprecipitation assay (RIPA) lysis buffer was purchased from Santa Cruz Biotechnology (Dallas, TX, USA). All antibodies except for antifibronectin antibody (Abcam, Cambridge, MA, USA) were purchased from Cell Signaling Technology (Beverly, CA, USA).

Cell culture. The permanent cell line, SK-HEP-1, is a hepatic adenocarcinoma line derived from liver sinusoidal endothelial cells. This cell line was obtained from the American Type Culture Collection (ATCC, Wiltshire, USA). SK-HEP-1 cells were maintained in Minimum Essential Medium (Gibco, Carlsbad, CA, USA) with $10 \%$ fetal bovine serum (Gibco), 100 units $/ \mathrm{mL}$ penicillin, $100 \mu \mathrm{g} / \mathrm{mL}$ streptomycin (Invitrogen, Carlsbad, CA, USA), and $100 \mu \mathrm{M}$ non-essential amino acids (Gibco) in a humidified atmosphere containing $5 \% \mathrm{CO}_{2}$ at $37^{\circ} \mathrm{C}$.

Cell-cycle analysis. Following treatment, cells were washed twice with phosphate-buffered saline (PBS; Invitrogen, Carlsbad, CA, USA) and were then fixed in $70 \%$ ethanol overnight at $-20^{\circ} \mathrm{C}$. Fixed cells were stained with a solution containing $10 \mu \mathrm{g} / \mathrm{mL}$ of propidium iodide (PI; Sigma, St. Louis, MO, USA), $100 \mu \mathrm{g} / \mathrm{mL}$ of RNase A (Sigma) and $0.1 \%$ Triton X-100 (Sigma) at $37^{\circ} \mathrm{C}$ in the dark. After 30 min, cell cycle distribution was performed using FACScan (BD Biosciences, Bedford, MA, USA) flow cytometry.

Cell viability, migration and invasion assays. Cell cytotoxicity and viability were determined by measuring the activity of mitochondrial malate dehydrogenase (mMDH) using the WST-1 assay (13). Basically, cells were seeded in 24-well plates at $1 \times 10^{4}$ cells/well and were cultured in MEM containing $10 \% \mathrm{FBS}$ at $37^{\circ} \mathrm{C}$. Cell proliferation was determined at 24 hours after seeding by adding the WST-1 reagent and incubating the cells for 3 hours further.

The migration assay was performed using the Ibidi Culture-Insert (Ibidi GmbH, Munich, Germany) and Millicell 24-well hanging inserts with $8 \mathrm{~mm}$ PET membranes (EMD Millipore, Billerica, MA, USA). The Ibidi Culture-Insert was mainly developed to replace classical scratch assays. Silicon inserts, placed on a cell culture surface, provide two cell culture reservoirs. These two chambers are separated by a $500 \mu \mathrm{m}$ thick wall. For Millicell hanging cell culture inserts, the lower compartment was filled with MEM containing $20 \%$ FBS. Cells with CTAB treatment were placed in the upper part of the chamber and were incubated for 16 hours. Following incubation, the cells were fixed with methanol and stained with crystal violet for 1 hour. The cells on the upper surface of the filter were removed using a cotton swab. The filters were subsequently rinsed in distilled water 
A

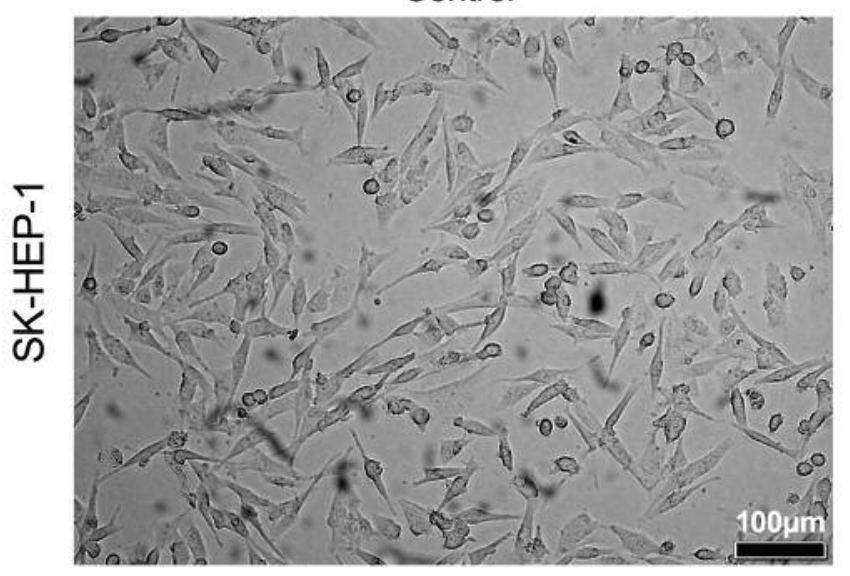

Control

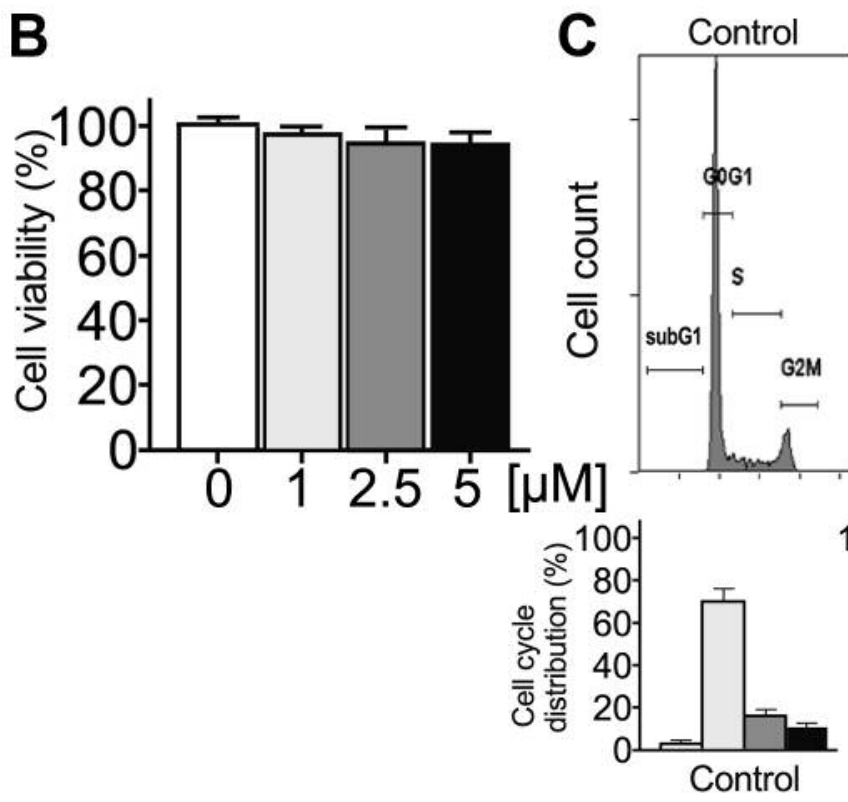

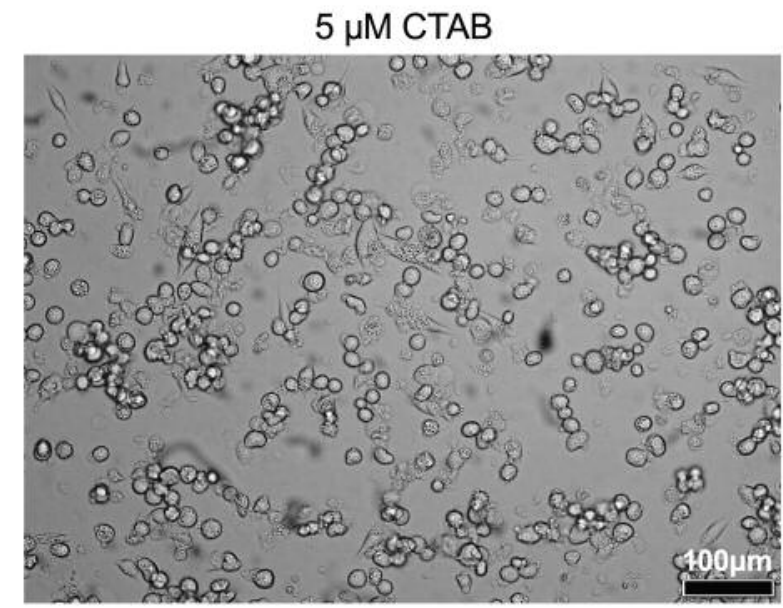
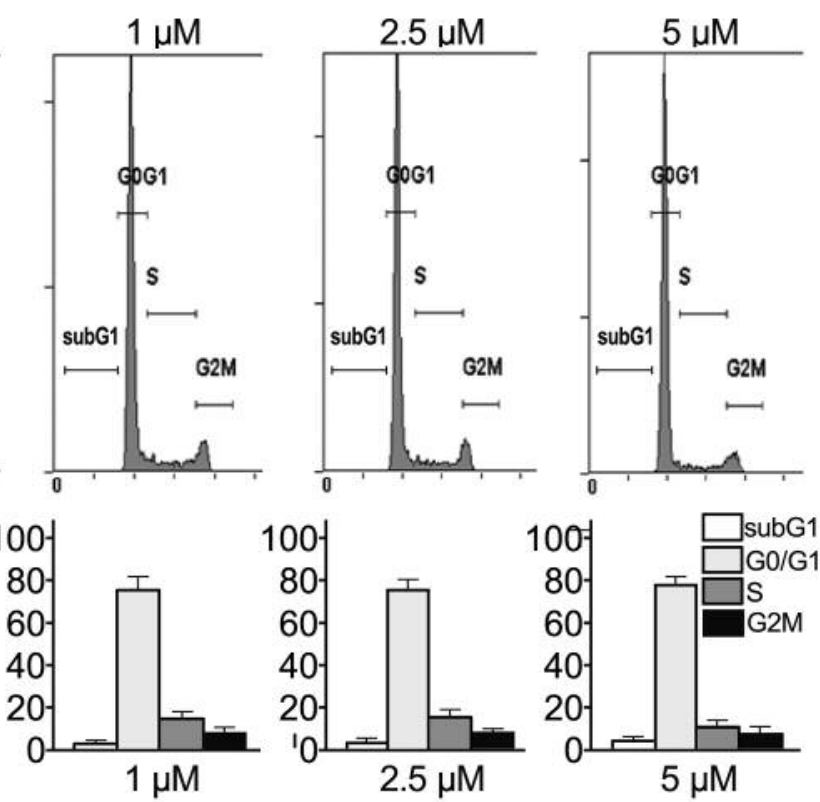

Figure 1. Morphological changes of CTAB-treated SK-HEP-1 cells. (A) Images captured using phase-contrast microscope revealed changes in morphology from spindle shaped (control) to cobblestone-like in SK-HEP-1 cells with CTAB treatment. Scale bars: $100 \mu \mathrm{m}$. (B) The effect of treatment with vehicle (control) or 1, 2.5 and $5 \mu \mathrm{M}$ of CTAB for $24 \mathrm{~h}$ was determined by the WST-1 assay. Absorbance values obtained from SK-HEP-1 cells treated with vehicle were taken as 100\%. (C) Flow cytometry of CTAB-treated SK-HEP-1 cells assessed their distribution within the cell cycle. Values are mean $\pm S E$ of the percentage of cells in the subG1 (white bars), $G_{0} / G_{1}$ (light gray bars), $S$ (dark gray bars) and $G_{2} / M$ (black bars) phases of the cell cycle. Data are representative of three independent experiments. *Statistically significant with $p<0.05$, when compared with the control group $(0 \mu M)$.

until no additional stain leaching was observed. The cells were then air-dried for 15 minutes. The migratory phenotypes were determined by counting the cells that migrated to the lower side of the filter through microscopy at $100 \times$ magnification. For each membrane, a total of 5 fields were selected at random and were counted, and each sample was assayed in triplicate experiments.

The invasion assay was performed using Millicell 24-well hanging inserts. The upper side was pre-coated with $10 \mu \mathrm{g} / \mathrm{mL}$ Matrigel (BD Biosciences, Bedford, MA, USA). Cells with CTAB treatment were placed in the upper part of the chamber and were incubated at $37^{\circ} \mathrm{C}$ for $24 \mathrm{~h}$. The experimental procedures were identical to the migration assay procedures.

Immunoblotting analysis. For Western blotting, cells were lysed in RIPA buffer containing protease and phosphatase inhibitors (Roche, Mannheim, Germany). Equal amounts of protein extracts were subjected to immunoblot following methods described previously (14).

Statistical analysis. The data are expressed as mean \pm standard errors (SE) of three independent experiments and were analyzed by one- 
way analysis of variance (ANOVA) using the GraphPad prism software program (Graph Pad Software, La Jolla, CA, USA). Student's t-test was used in two-group comparisons. $p<0.05$ was considered as statistically significant.

\section{Results}

$C T A B$ alters morphology but cannot affect growth and cell cycle distribution of CTAB-treated SK-HEP-1 cells. In order to evaluate the optimal dosage of CTAB to reverse EMT in SK-HEP-1 cells without affecting their proliferation and viability, the percentage of viable cells was determined using the WST-1 assay after cells were treated with $0,1,2.5$ and $5 \mu \mathrm{M}$ of CTAB for 24 hours. As shown in Figure 1A, vehicle-treated SK-HEP-1 cells displayed a fibroblast-like morphology. However, they showed a cobblestone-like morphology following treatment with $5 \mu \mathrm{M}$ CTAB for 24 hours. Our data also showed that CTAB-treated cells retained more than $90 \%$ cell viability at 24 hours (Figure 1B). Dosage of CTAB up to $5 \mu \mathrm{M}$ did not seem to have a cytotoxic effect on SK-HEP-1 cells. To determine whether CTAB affected cell cycle distribution of the CTAB-treated SK-HEP-1 cells, we performed FACS analysis at 24 hours following CTAB treatment. Cell cycle analysis showed that CTAB caused no alteration of the cell cycle progression in SK-HEP-1 cells (Figure 1C). Based on these results, CTAB has no effect on growth, apoptosis (the peak of sub-G1 on DNA content histogram), and cell cycle distribution in SK-HEP-1 cells.

$C T A B$ attenuates cellular migration and invasion of SK-HEP1cells. The distinct morphology evident in SK-HEP-1 cells treated with CTAB was reminiscent of cells that have undergone MET (Figure 1A). Accumulating evidence suggests that cellular migration and invasion are indispensable in tumor metastasis (5). Herein, both wound healing and transwell assays were performed to evaluate the role of CTAB in these processes. As shown in Figure $2 \mathrm{~A}$ and $\mathrm{B}$, the result of the wound healing and transwell migration assays showed that CTAB retarded significantly the migration of SK-HEP-1 cells. Next, the invasive potential of SK-HEP-1 cells, using the Matrigel-coated chamber, was further analyzed in the absence or presence of CTAB. The amount of SK-HEP-1 cells exposed to CTAB that moved through the membrane of the Matrigel chamber decreased about $83 \%$ compared to control cells (Figure 2C). In addition, CTAB reduced the migration ability of SK-HEP-1 cells by a $78 \%$ as compared to the control cells (Figure 2B). These findings indicate that CTAB diminishes the invasive capacity and migration ability of SK-HEP-1 cells.

$C T A B$ inhibits the protein expression of MMP-2 and MMP-9 in SK-HEP-1cells. Previous studies have shown that MMP-2 and MMP-9 play an important role in cancer metastasis through the degradation of the extracellular matrix (ECM) (15).
To examine whether CTAB regulates invasion by altering the expression of MMP-2 and MMP-9, immunoblotting analysis demonstrated that the protein levels of MMP-2 and MMP-9 were inhibited in SK-HEP-1 cells in response to CTAB treatment (Figure 3A). This result indicates that CTAB significantly down-regulates MMP-2 and MMP-9 expressions in SK-HEP-1 cells in a dose-dependent manner.

$C T A B$ increases the protein expression of TIMP-1 and TIMP2 in SK-HEP-1cells. TIMP-associated proteins are known to inhibit the enzymic activities and protein expression of MMPs $(16,17)$. The balance between the ratio of TIMPs and MMPs has been suggested to be a predictive marker of cancer metastasis $(17,18)$. We investigated whether CTABaltered MMPs were related to TIMPs. SK-HEP-1 cells were treated with $1,2.5$ and $5 \mu \mathrm{M}$ of CTAB for 24 hours, and TIMP-1 and TIMP-2 levels of their protein expression are shown in Figure 3A. Treatment of SK-HEP-1 cells with CTAB for 24 hours significantly increased the protein expression of TIMP-1 and TIMP-2. This result suggests that TIMPs signals may mediate inhibition of MMPs activities in CTAB-treated SK-HEP-1 cells.

$C T A B$ restrain the protein expression of Racl, cdc42 and RhoA in SK-HEP-1 cells. Due to the involvement of the small GTPases of the Ras homologous (Rho)-family (Rho-family small GTPases), including Rac1, cdc42 and RhoA proteins, in tumorigenesis, particularly in EMT/MET, migration/invasion, and metastasis (19), we assessed whether CTAB-altered cellular morphology was related to the small GTPases of the Rho family. Based on our Western blot data (Figure 3B), it was revealed that CTAB negatively affects the protein levels of Rac1, cdc42 and RhoA in SK-HEP-1 cells.

CTAB reverses EMT-associated biomarkers in SK-HEP-1 cells. Since morphological changes and EMT-associated protein-level alterations have been shown to be inter-

Figure 2. CTAB inhibits the migration and invasion of SK-HEP-1 cells. (A) A confluent culture of SK-HEP-1 cells was wounded by using the Ibidi Culture-Insert with the cells treated without or with indicated concentrations of CTAB. The wound was photographed with an inverted microscope. Scale bars: $200 \mu \mathrm{m}$. In vitro migration (B) and invasion (C) percentages of the $S K-H E P-1$ cell line upon treatment with CTAB are shown. SK-HEP-1 cells were treated with $5 \mu M$ CTAB. Following treatment, cells were allowed to migrate through uncoated transwell system for $16 h(B)$ or invade through a Matrigel-coated transwell system for $24 h(C)$. The number of cells at the bottom of the transwell filter was counted at the end of each assay. Results are presented as mean $\pm S E$ from three independent experiments, performed in duplicates, all versus control (cells treated with PBS, vehicle). Scale bars: $100 \mu \mathrm{m}$. $* * p<0.01$, when compared with the control group. 
A

Control

CTAB, $5 \mu \mathrm{M}$
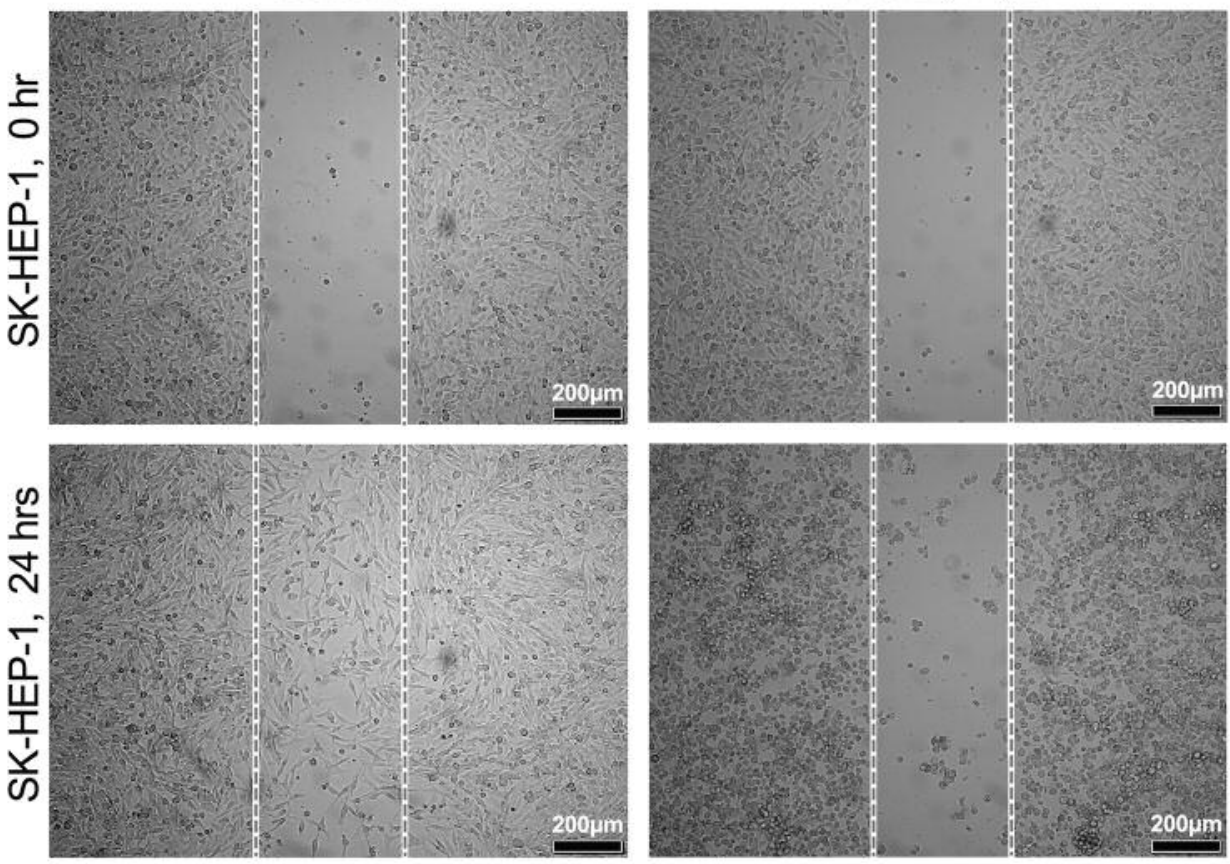

B
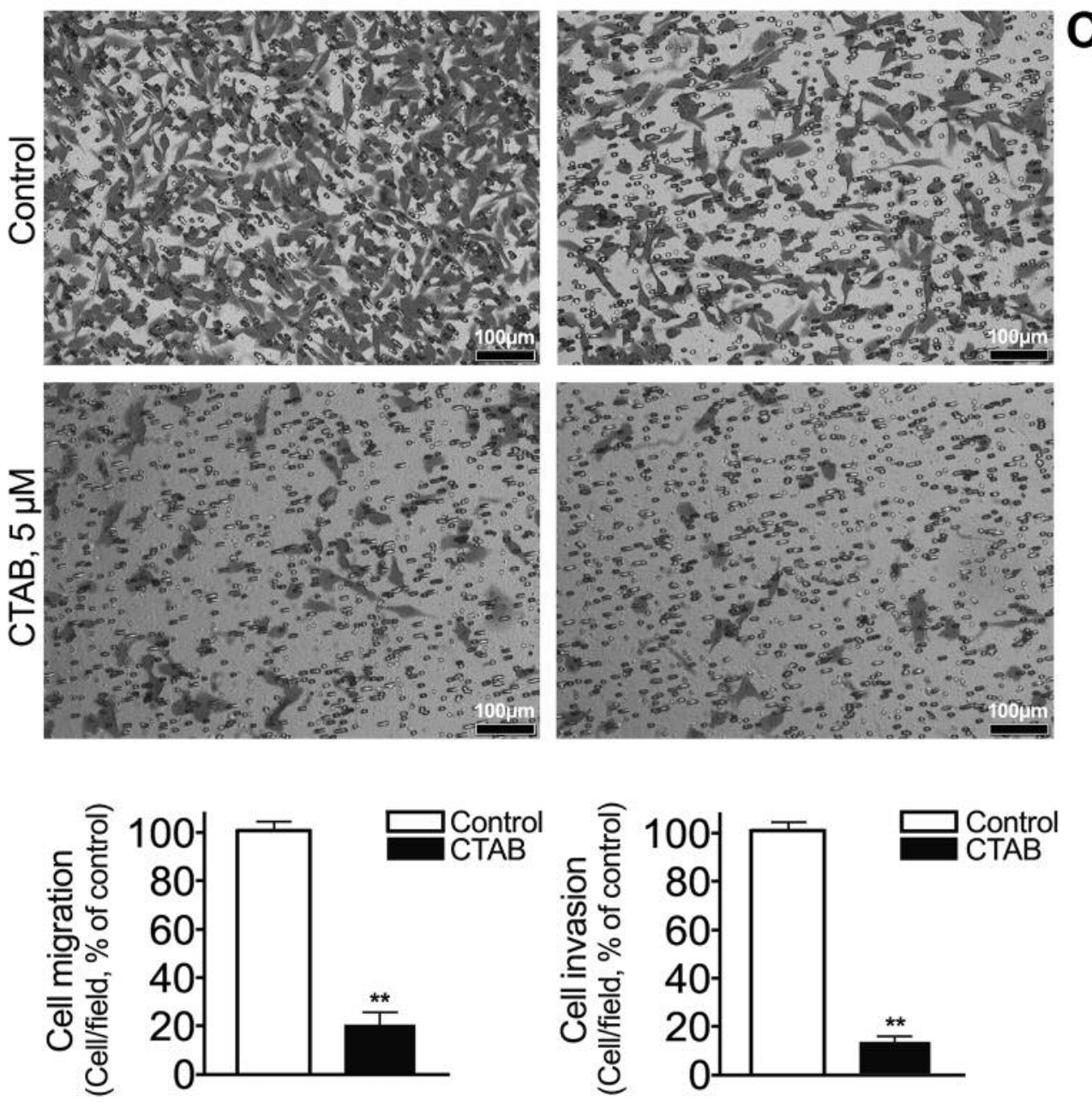


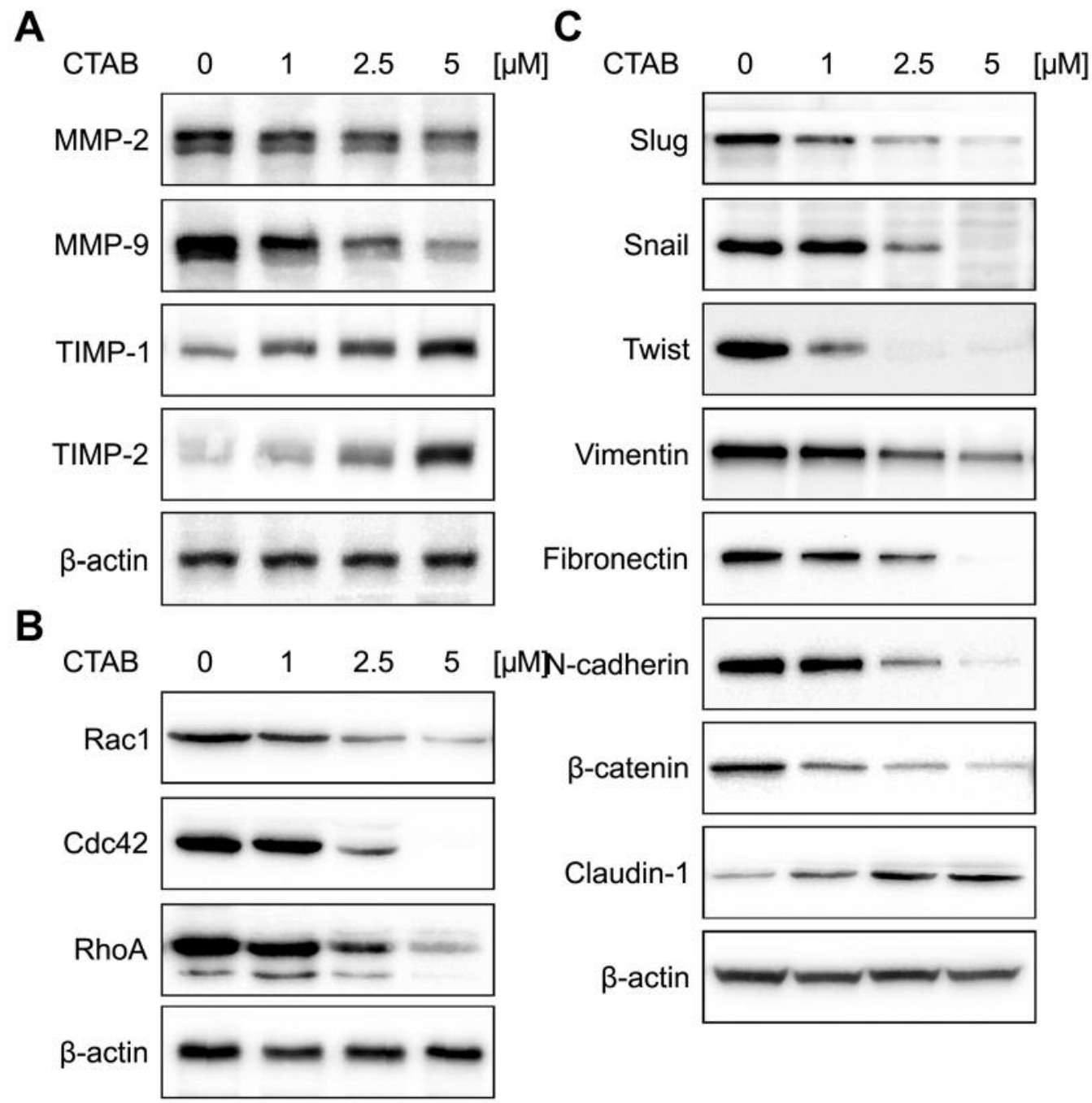

Figure 3. CTAB affects the levels of metastasis-related proteins in SK-HEP-1 cells. Immunoblotting analysis was used to analyze the expression levels of (A) MMP-2, MMP-9, TIMP-1 and TIMP-2, (B) Rac1, Cdc42 and RhoA, and (C) EMT-associated biomarkers in CTAB-treated SK-HEP-1 cells. Cells were treated with vehicle or increasing concentrations of CTAB (1 to $5 \mu M)$ for 24 h, and then whole-cell extracts were subjected to Western blot analysis. $\beta$-actin was used as a loading control. The results are representative of three independent experiments.

correlated (20), we studied the effect of CTAB on EMT in SK-HEP-1 cells. To confirm whether CTAB could affect EMT in SK-HEP-1 cells, cells were dosed in a range of 1 to $5 \mu \mathrm{M}$ CTAB. The expression levels of the EMT-related proteins were determined by Western blotting (Figure 3C). Our results showed that slug, snail, twist, vimentin, fibronectin, $\mathrm{N}$-cadherin, and $\beta$-catenin expression were decreased in a dose-dependent manner, while claudin-1 was increased in CTAB-treated SK-HEP-1 cells. Cells treated with CTAB displayed a decreased expression of mesenchymal markers concomitant with an increased expression of the epithelial marker (Figure 3C). When MET is in progress, the mesenchymal cells tend to have an epithelial morphology, hence the expression of mesenchymal markers decreases while the expression of epithelial markers increases. Both the morphological and molecular changes suggest that SK-HEP-1 cells might undergo reversal of EMT with exposure to CTAB.

Down-regulation of Smad/MAPK/PI3K/Akt/mTOR phosphorylation by $C T A B$ in $S K-H E P-1$ cells. In order to clarify the possible anti-EMT mechanism triggered by CTAB in SK-HEP1 cells, we further screened the expression levels of proteins involved in major signaling pathways involved in MET, including TGF- $\beta$, Wnt, Notch, Hedgehog and others (21). Here, we showed that CTAB significantly attenuated the 


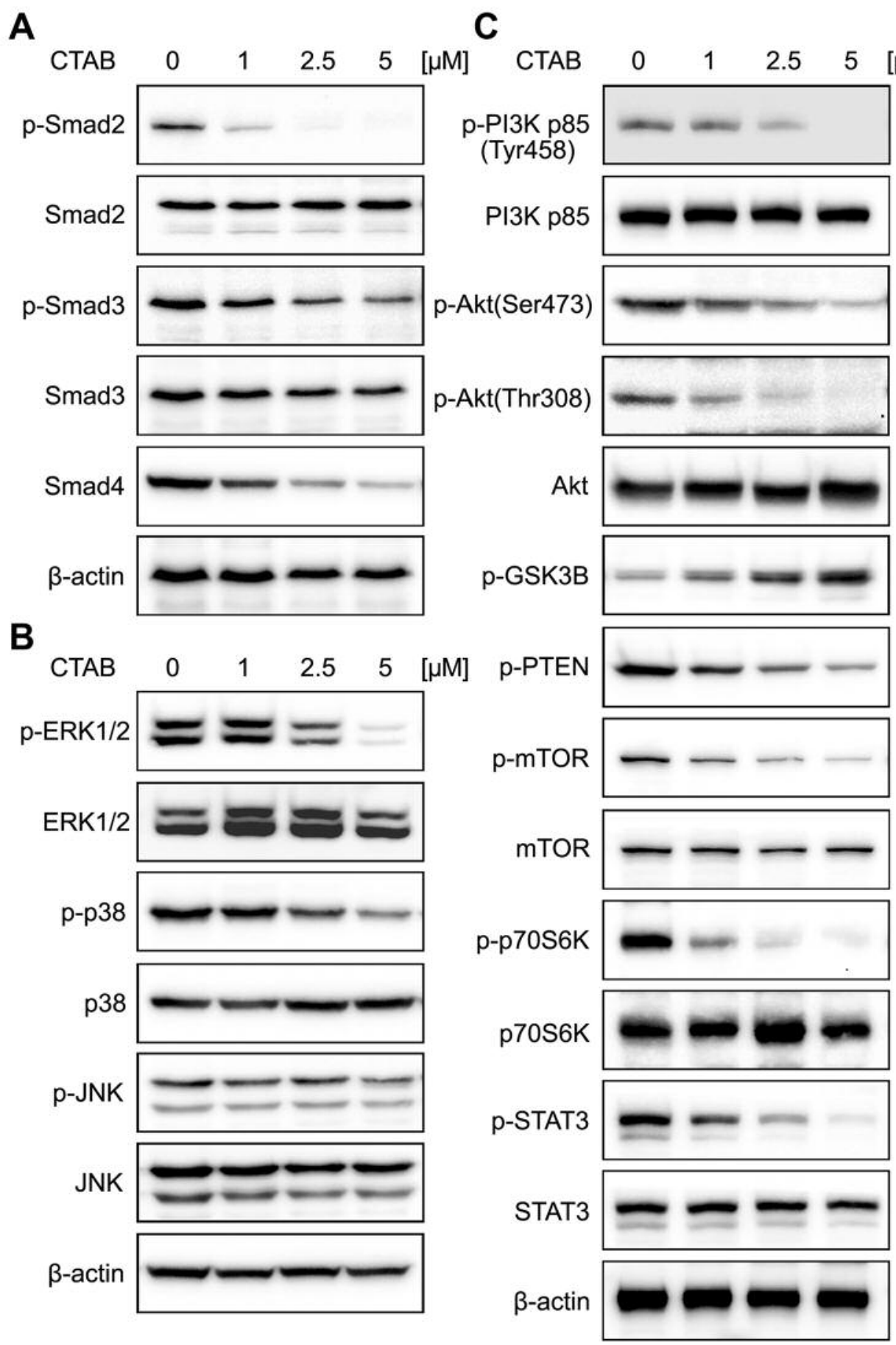

Figure 4. Effect of CTAB on expression of EMT-associated proteins in SK-HEP-1 cells. The expression levels of (A) Smad family, (B) MAPK family, (C) PI3K/Akt/mTOR-related pathway proteins were detected by Western blot analysis.

phosphorylation of Smad2 (downstream of canonical TGF- $\beta$ signaling pathway) (22), Smad3 (downstream of canonical TGF- $\beta$ signaling pathway) (22), ERK1/2, p38, JNK (ERK1/2, p38, JNK have been identified as three subfamilies of MAPKs)
(23), PI3K, Akt (downstream effector of PI3K and PTEN) (24), PTEN (PTEN is a negative regulator of PI3K-Akt signaling) (24), mTOR, p70S6K (downstream effector of mTOR) (25), STAT3 (downstream of TGF- $\beta$ signaling) (26) in SK-HEP-1 
cells (Figure 4). In contrast to the phosphorylated proteins, total levels of the cognate proteins, including Smad2, Smad3, ERK1/2, p38, JNK, PI3K, Akt, PTEN, mTOR, p70S6K, STAT3, were unaltered. By contrast, CTAB treatment markedly increased the phosphorylation of p-GSK3B levels in a concentration-dependent manner.

$T G F-\beta 1$ rescues the down-regulation of $S m a d 2 / 3, E R K 1 / 2$, p38, Akt, STAT3 phosphorylation signaling pathways by $C T A B$. Previous studies have reported that TGF- $\beta$ could influence EMT through various cellular messengers, including MAPK and PI3K pathways, via Smad-dependent and Smad-independent transcriptional mechanisms $(27,28)$. To verify the effect of CTAB on TGF- $\beta$ signaling pathway in SK-HEP-1 cells, cells pretreated with CTAB were subsequently incubated with TGF- $\beta 1$. Our data showed that the phosphorylation of $\mathrm{Smad} 2, \mathrm{Smad} 3$ and downstream signaling proteins, including Akt and GSK3B were rescued by the TGF- $\beta 1$ treatment (Figure $5 \mathrm{~A}$ ). Additionally, Smadindependent pathways, such as ERK1/2, p38, $\beta$-catenin and STAT3, were also rescued following treatment with TGF- $\beta 1$. We found that treatment with TGF- $\beta 1$ could sufficiently rescue the expression of mesenchymal markers, including slug, snail, twist, vimentin and fibronectin. These results show that CTAB exerts its anti-EMT effect by altering TGF$\beta$ signaling pathway in SK-HEP-1 cells. TGF- $\beta$ has also been shown to induce the expression of MMPs through Smad- and PI3K/Akt/mTOR-dependent pathways in liver cancer cells (29). Therefore, we assessed whether TGF- $\beta$ could rescue the effect of CTAB-reduced expression levels of MMP-2 and MMP-9 proteins. As shown in Figure 5A, CTAB-reduced the expression of MMP-2 and MMP-9 proteins that was rescued by TGF- $\beta$ signaling. In addition, retrieval of TGF- $\beta$ activity partially rescued the cellular morphology from cobblestonelike to spindle shaped (Figure 5B). Moreover, addition of TGF- $\beta 1$ also rescued migration and invasiveness (Figures $5 \mathrm{C}$ E). Taken together, these results suggested that CTAB modulates the EMT process via the canonical and noncanonical TGF- $\beta$ signaling pathways in SK-HEP-1 cells.

\section{Discussion}

Liver cancer is classified as a highly malignant tumor. Metastasis of liver cancer is related to poor prognosis and is a leading cause of death (30). Metastasis is a complex progression involving several stages, including invasiveness, intravasation, extravasation, and growth in other distant sites and organs. Tumor invasion across the ECM is thought to be one of the critical stages. The ability of tumor cells to degrade ECM components is associated with the presence of cells with metastatic potential (31). Accumulating evidence suggests that EMT plays an important role during the progression and metastasis of liver cancer, and the TGF- $\beta$ signaling pathway could act as a transcriptional regulator to mediate EMT (21). Hence, cancer patients are in urgent need of a novel anti-metastatic agent that targets EMT-associated pathways to bring benefit to those with metastatic liver adenocarcinoma.

In the present study, the cytotoxicity of CTAB was evaluated in the SK-HEP-1 cell line model. SK-HEP-1, a highly aggressive liver cancer cell line, was chosen as a cellular model to also assess the antitumoral effects of CTAB. First, we provided cell-based assays to determine whether CTAB could be an anti-metastatic agent against hepatic adenocarcinoma cell line. We found CTAB obviously promoted MET by morphological changes from spindle-like to cobblestone-like appearance of SK-HEP-1 cells after $24 \mathrm{~h}$. In addition, we demonstrated that CTAB attenuated the migratory capability and invasion capacity of the SK-HEP-1 cells, which involved the down-regulation of TGF- $\beta$-mediated Smad and PI3K/Akt/mTOR signaling pathways, while the attenuation of mesenchymal traits suggested an anti-metastatic effect. EMT can be identified by the morphological transformation of cells, the down-regulation of epithelial biomarkers, and the up-regulation of mesenchymal biomarkers and transcription factors (27). Additionally, several proteolytic enzymes, such as MMP-2 (gelatinase A) and MMP-9 (gelatinase B, type IV collagenase), are thought to be involved directly with the migration, invasion and metastasis of tumor cells, and are associated with patient prognosis, pathological grade, and clinical stage (32). In this study, the results from western blotting demonstrated that CTAB strongly reduced the levels of two members of the MMPs family. Moreover, CTAB-treatment resulted in decreased expression of mesenchymal markers in SK-HEP-1 cells in a dose-dependent manner. As these cells normally show an increased expression of mesenchymal markers in culture, it seemed that CTAB treatment caused the mesenchymal to epithelial switch by reducing the expression of mesenchymal markers. The results indicate that $\mathrm{CTAB}$ effectively reduces the migration and invasion of SK-HEP-1 cells.
Figure 5. CTAB down-regulated the proteins of TGF- $\beta /$ Smad and PI3K/Akt/mTOR-related pathways and affected EMT was rescued by $T G F-\beta 1$ in SK-HEP-1 cells. (A) Cells were pretreated with indicated concentrations of TGF- $\beta 1$ for $16 \mathrm{~h}$, and then they were co-treated with $C T A B$ for $24 h$. The levels of each protein expression were analyzed by western blotting. $(B)$ The cells pretreated with TGF- $\beta 1$ for $16 \mathrm{~h}$ were treated with CTAB for $24 \mathrm{~h}$ and then their morphological changes were examined using a microscope. Scale bars: $100 \mu m$. TGF- $\beta 1$ rescued the $C T A B$-inhibited motility as analyzed using wound healing $(C)$ and transwell system assays (D). Scale bars: $200 \mu \mathrm{m}$. (E) The TGF- $\beta 1$ rescued cellular invasiveness of CTAB-treated cells was examined under transwell system and the results are presented as a percentage of the control (100\%). **p<0.01, when compared with the control group. 
A
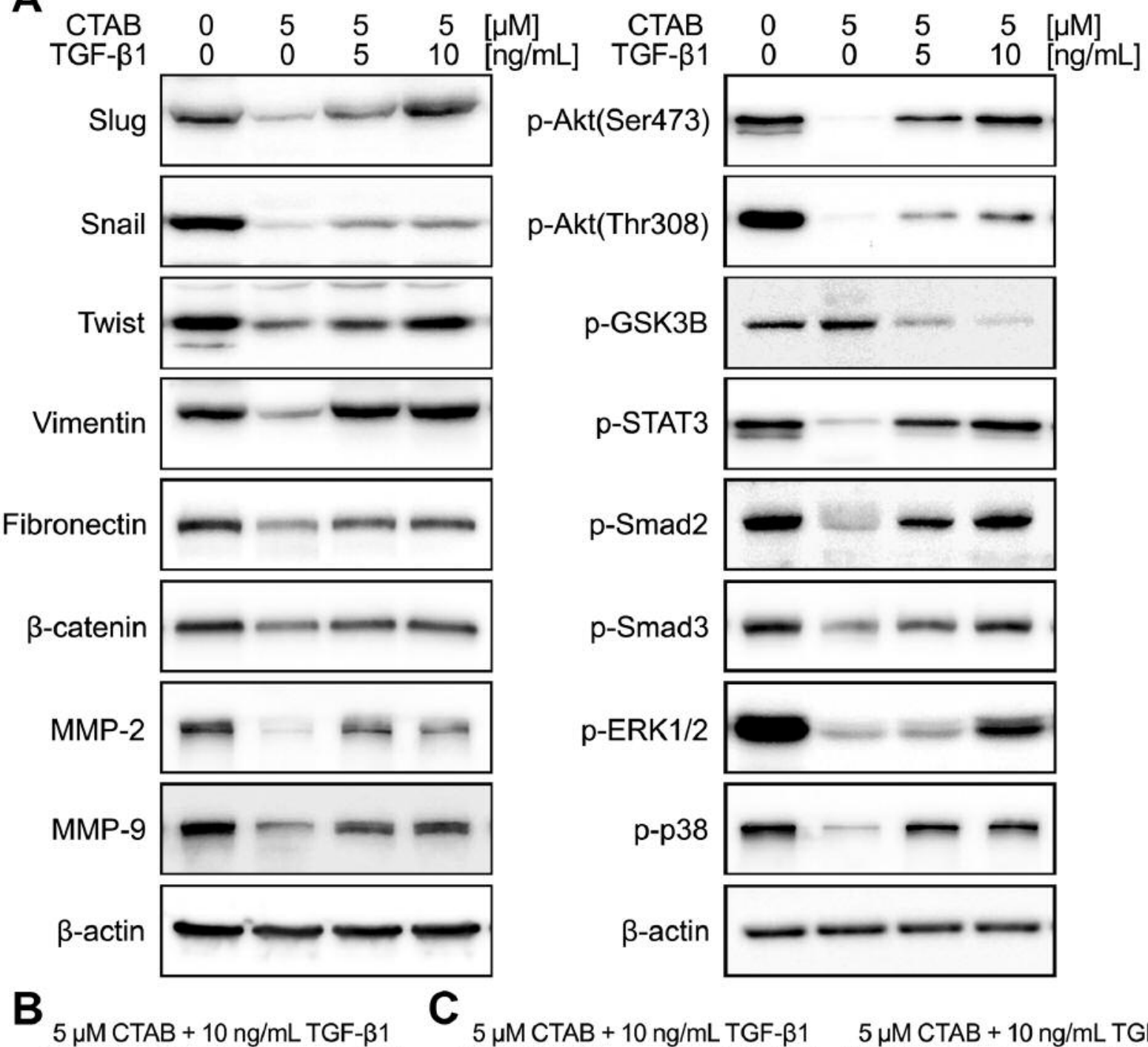

$5 \mu \mathrm{M}$ CTAB $+10 \mathrm{ng} / \mathrm{mL}$ TGF- $\beta 1 \quad 5 \mu \mathrm{M}$ CTAB $+10 \mathrm{ng} / \mathrm{mL}$ TGF- $\beta 1$
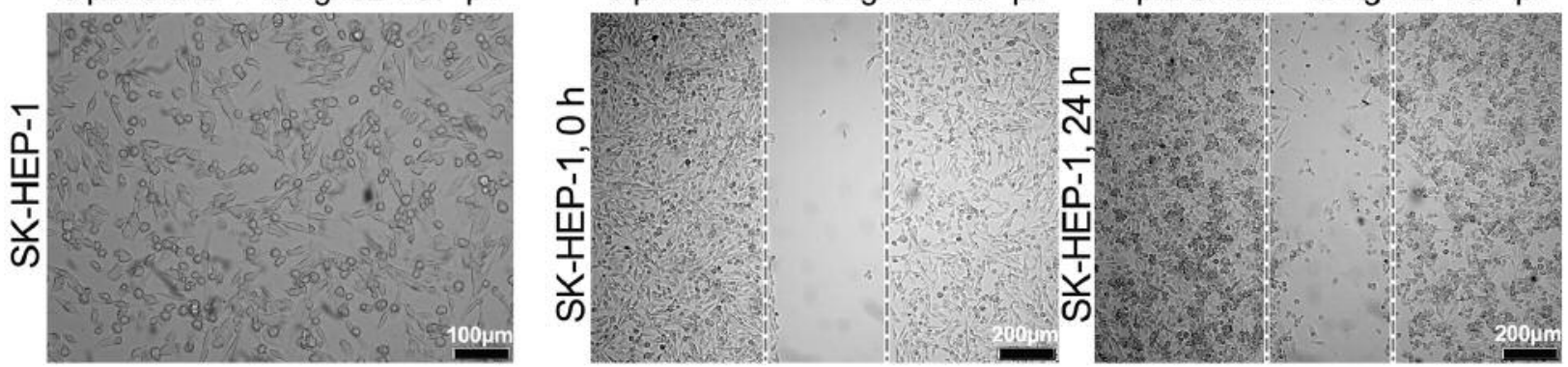

D
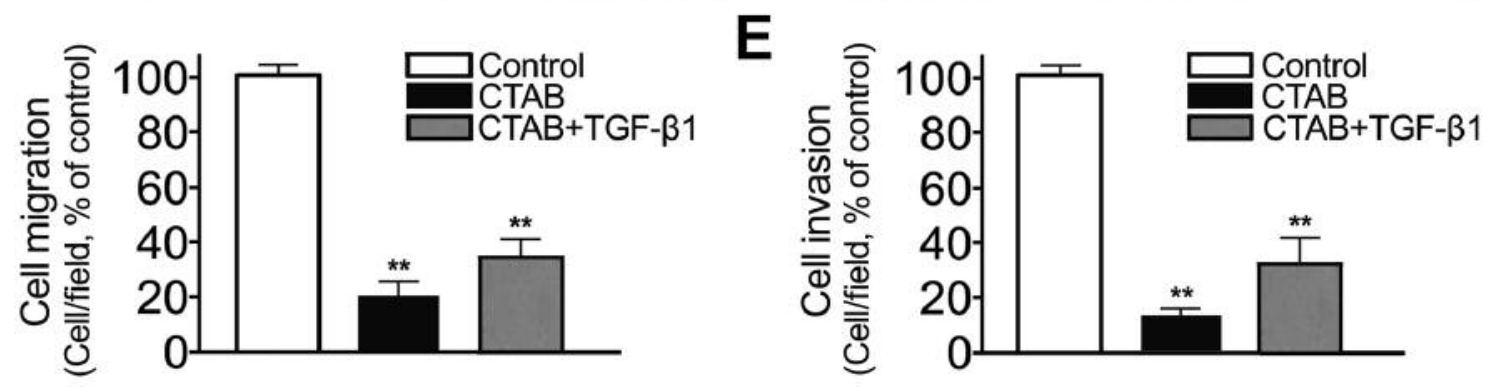
Experimentally-reduced EMT was then induced following exposure of SK-HEP-1 cells to CTAB or TGF- $\beta$ as evidenced by the modulation of the expression levels of EMT markers. Treatment with TGF- $\beta$ could rescue the MET phenotype, which allowed tumor cells to migrate and invade adjacent tissues. Tumor metastasis, including cell adhesion, proteolytic degradation of extracellular matrix (ECM) and cell migration, is critically associated with the acquisition of an EMT phenotype (33). Therapy for metastasis is based on blocking of one of these pathways (34). In addition, there is evidence that TGF- $\beta$ participates in EMT and mediates tumor metastasis, including abrogation of cell-cell adhesion, contribution to mesenchymal phenotypes and migratory and invasive capabilities of liver cancer cells (35). TGF- $\beta$ signaling is often attenuated during hepatotumorigenesis, but is retained during the malignancy of hepatocellular carcinoma cells (36). Therefore, TGF- $\beta$ could be a therapeutic target for the treatment of liver cancer. The TGF- $\beta$ ligands bind to its receptors and then activates downstream signaling pathways, including those mediated by Smad-dependent and Smad-independent pathways (such as Ras, PI3K/Akt/mTOR and MAPK) (28, 37-39). In this study, we demonstrated that the addition of CTAB down-regulated the phosphorylation levels of Smad2/3, PI3K p85/Akt/ $\mathrm{mTOR} / \mathrm{p} 70 \mathrm{~S} 6 \mathrm{~K}$ in SK-HEP-1 in a concentration-dependent manner. We then demonstrated the influence of CTAB on EMT by Western blotting, as evidenced by the downregulation of slug, snail, twist, vimentin, fibronectin, $\mathrm{N}$-cadherin and $\beta$-catenin, and the up-regulation of claudin1 compared to TGF- $\beta 1$ treatment. Therefore, we suggest that CTAB blocks the TGF- $\beta /$ Smad and PI3K p85/Akt/mTOR signaling pathways, thereby preventing the progression of the EMT process.

Overall, our in vitro findings suggest that CTAB restrained hepatic cancer cell migration and invasion, at least in part, by down-regulating the canonical and non-canonical TGF- $\beta$ signaling pathways and by reversing the EMT process. This is the first time that the anti-metastatic effect of CTAB as a potential inhibitor is demonstrated. We propose that CTAB could be further tested as an effective drug candidate against tumor invasion and metastasis of human hepatic cancer through suppression of TGF- $\beta$ signaling.

\section{Conflicts of Interest}

All Authors declare that they have no conflicts of interest.

\section{Authors' Contributions}

Conceptualization was by $\mathrm{CJ}$, funding acquisition by TKW and $\mathrm{CH}$, Methodology and validation by YRP, CWH and FMH. Writing theoriginal draft was by TKW and CJL, reviewing and editing by JYL and CJL. All authors read and approved the final manuscript.

\section{Acknowledgements}

This study was supported by the Research Fund of Tung's Taichung MetroHarbor Hospital (TTMHH-107R0024 and TTMHH-108R0015) and Chang Bing Show Chwan Memorial Hospital (RD107036).

\section{References}

1 Bray F, Ferlay J, Soerjomataram I, Siegel RL, Torre LA and Jemal A: Global cancer statistics 2018: GLOBOCAN estimates of incidence and mortality worldwide for 36 cancers in 185 countries. CA Cancer J Clin 68: 394-424, 2018. PMID: 30207593. DOI: $10.3322 /$ caac. 21492

2 Schon HT and Weiskirchen R: Immunomodulatory effects of transforming growth factor- $\beta$ in the liver. Hepatobiliary Surg Nutr 3: 386-406, 2014. PMID: 25568862. DOI: 10.3978/ j.issn.2304-3881.2014.11.06

3 Poisson J, Lemoinne S, Boulanger C, Durand F, Moreau R, Valla D and Rautou PE: Liver sinusoidal endothelial cells: Physiology and role in liver diseases. J Hepatol 66: 212-227, 2017. PMID: 27423426. DOI: 10.1016/j.jhep.2016.07.009

4 Heffelfinger SC, Hawkins HH, Barrish J, Taylor L and Darlington GJ: SK-HEP-1: a human cell line of endothelial origin. In Vitro Cell Dev Biol 28: 136-142, 1992. PMID: 1371504.

5 Guan X: Cancer metastases: challenges and opportunities. Acta Pharm Sin B 5: 402-418, 2015. PMID: 26579471. DOI: 10.1016/j.apsb.2015.07.005

6 Chaffer CL and Weinberg RA: A perspective on cancer cell metastasis. Science 331: 1559-1564, 2011. PMID: 21436443. DOI: $10.1126 /$ science. 1203543

7 Zhou P, Li B, Liu F, Zhang M, Wang Q, Liu Y, Yao Y and Li D: The epithelial to mesenchymal transition (EMT) and cancer stem cells: implication for treatment resistance in pancreatic cancer. Mol Cancer 16: 52, 2017. PMID: 28245823. DOI: 10.1186/ s12943-017-0624-9

8 Weiss MJ, Wong JR, Ha CS, Bleday R, Salem RR, Steele GD Jr and Chen LB: Dequalinium, a topical antimicrobial agent, displays anticarcinoma activity based on selective mitochondrial accumulation. Proc Natl Acad Sci USA 84: 5444-5448, 1987. PMID: 3474661. DOI: 10.1073/pnas.84.15.5444

9 Bleday R, Weiss MJ, Salem RR, Wilson RE, Chen LB, and Steele G, Jr: Inhibition of rat colon tumor isograft growth with dequalinium chloride. Arch Surg 121: 1272-1275, 1986. PMID: 3778199.

10 Yip KW, Mao X, Au PY, Hedley DW, Chow S, Dalili S, Mocanu JD, BastianuttoC, Schimmer A and Liu FF: Benzethonium chloride: a novel anticancer agent identified by using a cellbased small-molecule screen. Clin Cancer Res 12: 5557-5569, 2006. PMID: 17000693. DOI: 10.1158/1078-0432.CCR-06-0536

11 Giraud I, Rapp M, Maurizis JC and Madelmont JC: Synthesis and in vitro evaluation of quaternary ammonium derivatives of chlorambucil and melphalan, anticancer drugs designed for the chemotherapy of chondrosarcoma. J Med Chem 45: 2116-2119, 2002. PMID: 11985479.

12 Ito E, Yip KW, Katz D, Fonseca SB, Hedley DW, Chow S, Xu GW, Wood TE, Bastianutto C and Schimmer AD: Potential use of cetrimonium bromide as an apoptosis-promoting anticancer agent for head and neck cancer. Mol Pharmacol 76: 969-983, 2009. PMID: 19654225. DOI: 10.1124/mol.109.055277 
13 Galas RJ and Liu JC: Surface density of vascular endothelial growth factor modulates endothelial proliferation and differentiation. J Cell Biochem 115: 111-120, 2014. PMID: 23913753. DOI: $10.1002 /$ jcb. 24638

14 Lee CJ, Hsu LS, Yue CH, Lin H, Chiu YW, Lin YY, Huang CY, Hung MC and Liu JY: MZF-1/Elk-1 interaction domain as therapeutic target for protein kinase $\mathrm{C} \alpha$-based triple-negative breast cancer cells. Oncotarget 7: 59845-59859, 2016. PMID: 27542222. DOI: 10.18632/oncotarget.11337

15 Al Dhaheri Y, Attoub S, Arafat K, Abuqamar S, Viallet J, Saleh A, Al Agha H, Eid A and Iratni R: Anti-metastatic and antitumor growth effects of Origanum majorana on highly metastatic human breast cancer cells: inhibition of $\mathrm{NFKB}$ signaling and reduction of nitric oxide production. PLoS One 8: e68808, 2013. PMID: 23874773. DOI: 10.1371/journal.pone.0068808

16 Libra M, Scalisi A, Vella N, Clementi S and Sorio R: Uterine cervical carcinoma: role of matrix metalloproteinases (review). Int J Oncol 34: 897-903, 2009. PMID: 19287946. DOI: 10.3892/ijo_00000215

17 Han X, Zhang H, Jia M, Han G and Jiang W: Expression of TIMP3 gene by construction of a eukaryotic cell expression vector and its role in reduction of metastasis in a human breast cancer cell line. Cell Mol Immunol 1: 308-310, 2004. PMID: 16225775.

18 Yang CM, Hu TY and Hu ML: Antimetastatic effects and mechanisms of apo-8'-lycopenal, an enzymatic metabolite of lycopene, against human hepatocarcinoma SK-Hep-1 cells. Nutr Cancer 64: 274-285, 2012. PMID: 22211877. DOI: 10.1080/ 01635581.2012 .643273

19 Ungefroren H, Witte D and Lehnert $\mathrm{H}$ : The role of small GTPases of the Rho/Rac family in TGF-beta-induced EMT and cell motility in cancer. Dev Dyn 247: 451-461, 2018. PMID: 28390160. DOI: $10.1002 /$ dvdy.24505

20 Hay ED: An overview of epithelio-mesenchymal transformation. Acta Anatomica 154: 8-20, 1995. PMID: 8714286.

21 Liu X, Yun F, Shi L, Li ZH, Luo NR and Jia YF: Roles of signaling pathways in the epithelial-mesenchymal transition in cancer. Asian Pac J Cancer Prev 16: 6201-6206, 2015. PMID: 26434817. DOI: 10.7314/apjcp.2015.16.15.6201

$22 \mathrm{Zi} \mathrm{Z}$, Chapnick DA and Liu X: Dynamics of TGF- $\beta / \mathrm{Smad}$ signaling. FEBS Lett 586: 1921-1928, 2012. PMID: 22710166. DOI: $10.1016 /$ j.febslet.2012.03.063

23 Wada T and Penninger JM: Mitogen-activated protein kinases in apoptosis regulation. Oncogene 23: 2838-2849, 2004. PMID: 15077147. DOI: $10.1038 /$ sj.onc. 1207556

24 Carnero A and Paramio JM: The PTEN/PI3K/AKT pathway in vivo, cancer mouse models. Front Oncol 4: 252, 2014. PMID: 25295225. DOI: $10.3389 /$ fonc. 2014.00252

25 Hay N and Sonenberg N: Upstream and downstream of mTOR. Genes Dev 18: 1926-1945, 2004. PMID: 15314020. DOI: $10.1101 / \mathrm{gad} .1212704$

26 Svinka J, Mikulits W and Eferl R: STAT3 in hepatocellular carcinoma: new perspectives. Hepat Oncol 1: 107-120, 2014. PMID: 30190945. DOI: 10.2217/hep.13.7

27 Gui T, Sun Y, Shimokado A and Muragaki Y: The roles of mitogen-activated protein kinase pathways in TGF- $\beta$-induced epithelial-mesenchymal transition. J Signal Transduct 2012: 289243, 2012. PMID: 22363839. DOI: 10.1155/2012/289243

28 Gonzalez DM and Medici D: Signaling mechanisms of the epithelial -mesenchymal transition. Sci Signal 7: e8, 2014. PMID: 25249658. DOI: 10.1126/scisignal.2005189
29 Xing S, Yu W, Zhang X, Luo Y, Lei Z, Huang D, Lin J, Huang Y, Huang $S$ and Nong F: Isoviolanthin extracted from dendrobium officinale reverses TGF- $\beta 1$-mediated epithelial-mesenchymal transition in hepatocellular carcinoma cells via deactivating the TGF- $\beta /$ Smad and PI3K/Akt/mTOR signaling pathways. Int J Mol Sci 19: 1556, 2018. PMID: 29882900. DOI: 10.3390/ijms19061556

30 Budhathoki N and Shah BK: Survival of elderly patients with advanced hepatocellular carcinoma with distant metastasis in pre- and post-sorafenib era: A population based study. J Clin Oncol 35: e15643, 2017. DOI: 10.1200/JCO.2017.35.15_ suppl.e15643

31 Cavallaro U and Christofori G: Cell adhesion in tumor invasion and metastasis: loss of the glue is not enough. Biochim Biophys Acta 1552: 39-45, 2001. PMID: 11781114. DOI: 10.1016/s0304419x(01)00038-5

32 Chambers AF and Matrisian LM: Changing views of the role of matrix metalloproteinases in metastasis. J Natl Cancer Inst 89: 1260-1270, 1997. PMID: 9293916. DOI: 10.1093/jnci/89.17.1260

33 Hanahan D and Weinberg RA: Hallmarks of cancer: The next generation. Cell 144: 646-674, 2011. PMID: 21376230. DOI: 10.1016/j.cell.2011.02.013

34 Steeg PS and Theodorescu D: Metastasis: a therapeutic target for cancer. Nat Clin Pract Oncol 5: 206-219, 2008. PMID: 18253104. DOI: $10.1038 /$ ncponc 1066

35 Malfettone A, Soukupova J, Fernando J, Koudelkova P, Bertran E, Fabra A, Grubinger M, Rani B, Giannelli G and Mikulits W: Crosstalk between TGF- $\beta$-induced epithelial-mesenchymal transition and stemness in hepatocellular carcinoma. J Hepatol 64: S557, 2016. DOI: 10.1016/S0168-8278(16)01003-5

$36 \mathrm{Mu}$ X, Lin S, Yang J, Chen C, Chen Y, Herzig MC, Washburn K, Halff GA, Walter CA, Sun B and Sun LZ: TGF-beta signaling is often attenuated during hepatotumorigenesis, but is retained for the malignancy of hepatocellular carcinoma cells. PLoS One 8: e63436, 2013. PMID: 23704908. DOI: 10.1371/journal.pone. 0063436

37 Croxford KP, Reader KL and Nicholson H: The potential role of transforming growth factor beta family ligand interactions in prostate cancer. AIMS Mol Sci 4: 41-61, 2017. DOI: 10.3934/ molsci.2017.1.41

38 Khan S, Shukla S, Sinha S, Lakra AD, Bora HK and Meeran SM: Centchroman suppresses breast cancer metastasis by reversing epithelial-mesenchymal transition via down-regulation of HER2/ERK1/2/MMP-9 signaling. Int J Biochem Cell Biol 58: 116, 2015. PMID: 25448414. DOI: 10.1016/j.biocel. 2014. 10.028

39 Li NY, Weber CE, Wai PY, Cuevas BD, Zhang J, Kuo PC and Mi Z: An MAPK-dependent pathway induces epithelial mesenchymal transition via Twist activation in human breast cancer cell lines. Surgery 154: 404-410, 2013. PMID: 23889968. DOI: $10.1016 /$ j.surg.2013.05.012
Received May 20, 2019

Revised June 18, 2019

Accepted June 20, 2019 